\title{
Slowing the opioid analgesic overdose epidemic
}

\author{
José G Merino US research editor, BMJ
}

\begin{abstract}
According to the Centers for Disease Control and Prevention (www.cdc.gov/mmwr/preview/mmwrhtml/mm6043a4.htm), more people in the United States overdose and die from prescription drug opioids than from cocaine and heroin combined. In 2008 alone, opioid analgesics were involved in 14800 deaths.
\end{abstract}

Several stakeholders, including federal and state governments, insurers, physicians and other health care providers, community organizations, and patients and their families, are taking steps to stop this epidemic. Recently, in the BMJ, Michael McCarthy described how state agencies and a health plan in the state of Washington are implementing policies that make it harder for physicians to prescribe ineffective high doses of long acting opioids, and for patients to "shop around" for doctors willing to prescribe narcotics, while minimizing the risk that patients will become addicted or divert their medications for use by others (BMJ 2012;345; e8340). Federal and local governments are joining the fight. This week, an advisory panel to the FDA recommended new restrictions on how drugs containing hydrocodone- the most frequently prescribed opioid analgesic - are prescribed (www.nytimes.com/2013/01/26/ health/fda-vote-on-restricting-hydrocodone-products-vicodin. html?ref=health\&_r=3\&). A few days ago, Mayor Bloomberg announced that in New York City, public hospitals will limit the doses that can be prescribed in the emergency room (www. nytimes.com/2013/01/11/nyregion/new-york-city-to-restrictpowerful-prescription-drugs-in-public-hospitals-emergencyrooms.html?ref=health). Critics of "legislative medicine" warn that these measures may not be effective and that they negatively impact on the ability of doctors to practice medicine. Drug companies and pharmacies may oppose new regulations.
Research will have to show whether these interventions have a significant effect on the incidence of opioid overdoses and deaths, and are worth the effort and political costs.

A complementary approach to reduce deaths is to intervene rapidly in overdose cases. Community based overdose prevention programs in 15 states and the District of Columbia promote the use of naloxone hydrochloride by bystanders to reverse the respiratory depression of opioid overuse. Data from CDC suggest that these interventions are effective; since 1996, these programs have trained over 53000 potential overdose bystanders (drug users, friends, family members, healthcare providers and staff at homeless shelters, substance abuse treatment programs, and prisons), and they report 10171 overdose reversals (www.cdc.gov/mmwr/pdf/wk/mm6106.pdf). But rigorous research supporting the value of this intervention has been lacking.

This week in the $B M J$, Alexander Walley and colleagues from Boston University report the impact of an overdose education and intranasal naloxone distribution (OEND) program sponsored by the Massachusetts Department of Health (doi:10.1136/bmj. f174). Between 2002 and 2009, these programs trained 2912 potential bystanders, who reported 327 rescues. Walley and colleagues found that in communities where the program was implemented, opium overdose rates fell significantly. Their results suggest that these programs are an effective intervention; future studies will have to determine whether they are cost-effective.

Cite this as: BMJ 2013;346:f730

๑ BMJ Publishing Group Ltd 2013 